\title{
Optical Stealth Design of Satellite
}

\author{
Dongjun Zhu' ${ }^{1}$, Zhanyue Zhang ${ }^{2}$, Letian Zheng ${ }^{1}$ \\ ${ }^{1}$ Department of Graduate Management, Equipment Academy, Beijing 101416, China \\ ${ }^{2}$ Department of Space Command, Equipment Academy, Beijing 101416, China
}

Keywords: Optical stealth, Optical visibility, Space surveillance.

\begin{abstract}
Satellites play an irreplaceable role in human daily life, more and more satellites are being sent into space. At the same time, foreign space powers are competing to develop the satellite stealth technology, the safety factors and survival ability of the satellite can't be ignored. Analysis of space surveillance system for high orbit satellite has a strong optical detection capability; optical visibility of spatial objects; and the optical brightness of the typical satellite body. Finally, it is pointed out that the development of satellite optical stealth technology is imperative.
\end{abstract}

\section{Introduction}

With the development of science and technology, satellite plays a huge role and the military power in civil and military fields, it is vigorously developing space target surveillance and stealth technology in the world. The United States Vandenberg launch team has successfully launched a space-based space surveillance satellite on September 25, 2010. This is the first satellite to be able to detect and track space targets from space[1]. At the same time the United States has also stepped up research on stealth satellites, military enterprises Lockheed Martin under the support of " misty " plan, from the beginning of 1980 has been vigorously developing stealth satellites, until now has been developed the third generation stealth satellites[2]. The above facts show that the space resources for the increasingly intensified, the satellite space security can't be ignored.

The safety factor of the satellite space has been one of the important factors in the process of research and development. The space safety factors from the ground detection and space-based detection. There are three ways to detect the satellite, which are radar, optical and passive tracking and positioning[3]. Among them, the detection accuracy of the optical system is higher, and the observation distance is further. It can effectively detect the high orbit satellite. The satellite itself is not luminous, so Space-Based Surveillance System of satellite detection, rely on the reflected light from the satellite surface brightness value, and its direct impact factors is the optical scattering characteristics of satellite surface, to reduce the optical detection capability of the other space surveillance system or to produce false judgment to realize the optical stealth, and improve the ability to survive.

\section{Optical probe source analysis}

Space target surveillance system is divided into two kinds of space-based and ground-based, keep track of on orbit service, stand-by and abandoned spacecraft, space debris and natural objects. The United States in the field of space target surveillance leading level, the ground-based equipment distribution in 16 locations around the world, from 20 different types of more than 30 sets of radio detectors, optical detector space-based detector and two control center. Ground optical equipment is not limited by the size and power, with the characteristics of long distance detection and high spatial resolution. Ground Space Surveillance Network (SSN) plays an important role in the United States surveillance system [4].

Ground space target monitoring system is vulnerable to weather, geographical position and time and other factors, not all weather to observe the space target. To overcome these shortcomings of the foundation system, in 2002 the United States began to start the space-based 
space surveillance system (SBSS) [5] can improve of space target detection, tracking and recognition ability, especially for high orbit satellite observations. It is said that the SBSS system will enable the United States to improve the ability to observe the earth orbit satellite $50 \%[6]$. SBSS system is composed of 4-8 satellites in low earth orbit optical remote sensing satellite constellation, the height of $1100 \mathrm{~km}$ and designed for a service life of 5 years, successful deployment can be realized after the day of space target surveillance a and update the most satellite location data [7]. It has the ability to observe the orbit objects and can observe the whole day, and the observation period is shorter, especially for the deep space objects. The SBSS is subject to size constraints, the future development of space target surveillance system is likely to become the space based, supplemented by the architecture foundation. The SBSS will play an irreplaceable role, not only can accurately estimate the potential rivals, spatial ability, but also the orbit of space target prediction, for possible collisions and on space target will be subject to attack notice etc.

\section{Optical stealth of satellite}

Optical stealth is a special term used to describe the "reduction of the optical signature of the target". In principle, it is consistent with the optical radiation characteristics of the target and the background, which makes the optical equipment can not identify the target from the background or generate false judgment, that is, to reduce the observation brightness of the satellite surface [9]. On the target star to take certain measures, within the maximum limit to reduce the probability of the target satellite is optical device discovery and exploration, is to achieve the satellite optical invisibility is improving satellite their ability to survive effective way [10]. The realization of optical stealth can be analyzed from three aspects, such as the shape design, material selection and occlusion interference.

\subsection{Shape design}

The shape and structure of space target of reasonable design, within the maximum reduce the energy of electromagnetic wave, wave infrared and visible light reflectivity or emissivity, which can "t be detected, or reduce the data validity to stealth purposes. For space-based observation system, observation to observe the existence of observation of a "dead", using geometry design will reflect sunlight to high orbit and observation "dead", reflected light optical system will accept very little to support optical devices to work normally. The satellite designed into space junk or hidden payload in order to reduce the other side of the satellite observation and judgment error. Spherical design will reflect a part of the sun's light to the earth's direction, and thus will be observed star detection, should be considered as far as possible to replace the smooth surface of the surface, and mirror reflection instead of diffuse reflection.

\subsection{Material selection}

Satellite surface material properties are one of the important factors affecting the optical properties of the satellite, so it can choose the suitable material to achieve the low brightness of the satellite surface from the point of view of material selection. The choice of materials is often combined with radar wave, infrared, visible light and so on. It is very difficult to obtain a wide range of applications. At present, the study of the satellite surface low brightness material is: coated multi band materials, thin film type multi spectral material, wide band energy absorption camouflage blanket, doped semiconductor materials and nanometer material [11].

The solar cell surface using suede design can not only reduce the reflectance of a surface of the battery, but also inside the battery formation trap light, so as to improve the photoelectric conversion efficiency. As shown in Figure 1, the light reflected by many times in the surface structure, the path relative to the plane structure longer. The reflection from the lower surface of the light, it is possible to fully reflect the angle of the impact of the battery surface. 


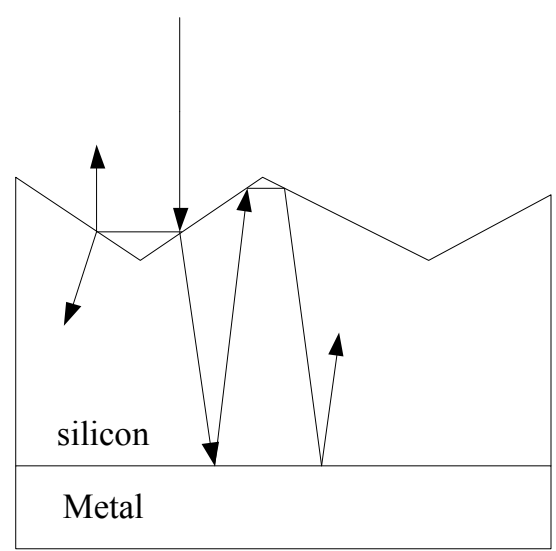

Figure 1 the surface structure of the incident light and reflected light sketch map

Taking silicon as an example, the refractive index of Silicon is 3.5, the critical angle of the total reflection of light is 16.6 degrees, and only the photon that is less than 16.6 degrees will not occur. So most of the photons will be absorbed, otherwise they will be reflected many times, eventually to the surface of the impact of less than 16.6 degrees from the surface of the solar cell. The smooth surface of the silicon solar cell reflectance is greater than 0.3 , and the light after cell knap surface structure in multiple reflections and refractions, reflectance of the surface of solar cells will be able to down to 0.1 , if plating reflective film and reflectivity can drop to about 0.03 [12]. By selecting the appropriate material, not only can achieve the purpose of optical stealth, but also improve the efficiency of photoelectric conversion.

\subsection{Occlusion interference}

Satellite features are installed on the satellite to suppress the mask and its auxiliary device, the purpose is to suppress the satellite laser, radar, visible and infrared characteristics, the Space-Based Surveillance System to detect satellite; or change the external characteristics of the satellite, the space-based surveillance system cannot separate it from the similar bait region. The exterior camouflage of the target satellite is mainly to consider the optical stealth, so as not to expose the payload information.

\section{Optical stealth computing model}

\subsection{Optical visibility analysis}

The main task of the Space-Based Surveillance System in optical equipment is of space target to tracking and orbit determination, to achieve the effective observation of space target, in the shadow of the earth, earth's shadow and sunlight conditions and other factors need to be considered.

(1)Earth shielding condition

In the light of the space is spread along a straight line, to ensure the effective observation of optical equipment, the connection between the observation satellite and the target satellite could not under the shadow of the earth, or the observation satellite and satellite is visible.

Among them, $R_{E}$ for the radius of the earth, $r_{o}, r_{p}, r_{1}$ for the earth's core to the target satellite $\mathrm{O}$, and the observation of the star $\mathrm{P}$ and the distance between $\mathrm{P}$ and $\mathrm{O}, \mathrm{h}$ for the edge of the high PO.

$h_{o}>R_{E}$, Geometric visible $h_{o}=r_{o} \sin \alpha$

$$
G_{\text {geometry }}=\left\{r_{o}, r_{\mathrm{p}}, r_{1} \mid r_{o} \sin \alpha>R_{E}\right\}
$$

(2)Earth shadow condition

When the satellite is running into the shadow area of the earth, the satellite can not be irradiated by the sun's light, and the optical device can not observe the target star. 
The included angle between the vector $\boldsymbol{r}_{\mathrm{o}}$ of the target satellite and the sun's center vector $\boldsymbol{r}_{\boldsymbol{s}}$ is $\beta$, the visible region of the target star under the earth shadow condition is $G_{\text {Shadow }}$

$$
G_{\text {Shadow }}=\left\{\boldsymbol{r}_{\boldsymbol{s}}, \boldsymbol{r}_{\boldsymbol{o}} \| \boldsymbol{r}_{\boldsymbol{o}} \mid \sin \beta>R_{E}\right\}
$$

(3)Sunlight condition

Satellite to the non imaging optical on the surface of optical device forming stray radiation, will reduce the satellite's observation ability, serious when the target image can be stray radiation noise and lead to optical equipment to normal work [13]. To achieve the observation of the target satellite, optical equipment must avoid direct area of solar light, the sun is not in the field of view of the optical device.

The included angle between the vector $\boldsymbol{r}_{P O}$ of the target satellite $\mathrm{O}$ relative to the observed satellite $\mathrm{P}$ and the vector $\boldsymbol{r}_{P S}$ of the sun $\mathrm{S}$ is $\theta$, The effective observation of optical equipment needs to meet the sum of $\theta$ larger than a certain critical angle of $\theta_{0}$, which is the sum of the visual radius and the scattering angle of the sun, The visible area is $G_{\text {Sunlight }}$

$$
G_{\text {Sunlight }}=\left\{\boldsymbol{r}_{\mathrm{po}}, \boldsymbol{r}_{\mathrm{ps}} \mid \theta>\theta_{0}\right\}
$$

\subsection{Optical brightness computing model}

The surface of the target satellite is composed of a large number of micro surface elements. The bidirectional reflectance distribution function of micro $d_{A}$ in the visible band of solar radiation $f_{r}$, $\mathrm{d}_{A}$ unit normal vector is $O N$, the unit vector $\mathrm{d}_{A}$ to the sun for $O S$ element, the unit vector of the $\mathrm{d}_{A}$ pointing detection system is $\boldsymbol{O D}$, The angle between the projection element $\boldsymbol{O} \boldsymbol{S}^{\prime}$ and $\boldsymbol{O} \boldsymbol{D}^{\prime}$ is $\psi$, the included angle between $\boldsymbol{O N}$ and $\boldsymbol{O S}$ is $\theta_{\mathrm{i}}$, the included angle between $\boldsymbol{O N}$ and $\boldsymbol{O D}$ is $\theta_{\mathrm{i}}$,

$$
\left\{\begin{array}{c}
\cos \theta_{\mathrm{i}}=\mathbf{O N} \cdot \mathbf{O S}, \quad \cos \theta_{r}=\mathbf{O N} \cdot \mathbf{O D} \\
\cos \psi=(\mathbf{O N} \times \mathbf{O S} \times \mathbf{O N}) \cdot(\mathbf{O N} \times \mathbf{O D} \times \mathbf{O N})
\end{array}\right.
$$

Definition of OCS of visible light scattering cross section of space target:

$$
\text { OCS }=\int_{A} f_{\mathrm{r}}\left(\theta_{\mathrm{i}}, \theta_{\mathrm{r}}\right) \cos \theta_{\mathrm{i}} \cos \theta_{\mathrm{r}} d A
$$

For the standard lambertian, $f_{\mathrm{r}}$ as a constant. take the ball as an example, establish OCS model.

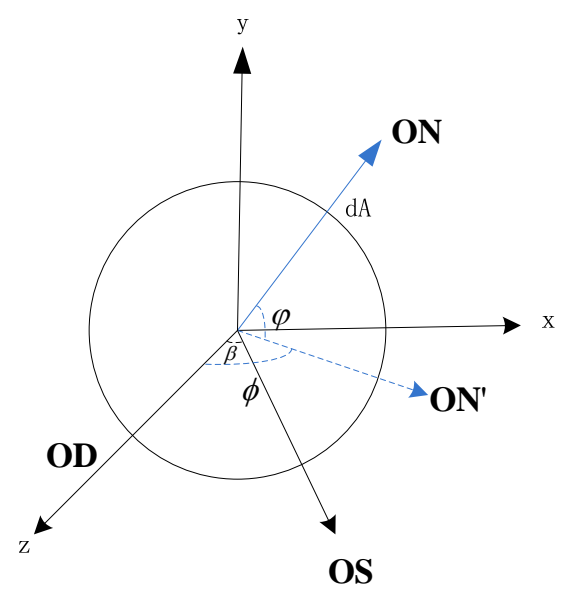

Figure 2 sphere and computational coordinate system

Integral infinitesimal element $\mathrm{d}_{A}$, Unit normal vector element $\boldsymbol{O N}$, The included angle between $\boldsymbol{O N}$ and planes $\mathrm{XOZ}$ is $\varphi, O N$ in the $\mathrm{XOZ}$ plane projection $\boldsymbol{O} \boldsymbol{N}^{\prime}$ and $\boldsymbol{O D}$ of the included angle is $\phi$. Detectors can be observed in the range of $\phi$ is $(-\pi / 2, \pi / 2)$, and the range of $\phi$ that the sun's light can 
illuminate is $(\beta-\pi / 2, \beta+\pi / 2)$. Therefore, the integral range of $\phi$ is $(\beta-\pi / 2, \pi / 2)$, and the integral range of $\varphi$ is $(-\pi / 2, \pi / 2)$. According to the vector product formula:

$$
\left\{\begin{array}{l}
\cos \theta_{\mathrm{i}}=\boldsymbol{O D} \cdot \boldsymbol{O N}=\cos \phi \cos \varphi \\
\cos \theta_{\mathrm{r}}=\boldsymbol{O S} \cdot \boldsymbol{O N}=\cos \varphi \cos (\beta-\phi)
\end{array}\right.
$$

\section{Stealth shape simulation}

Detector of the target satellite optical detection, by receiving the satellite reflection light to judge and analyze, the obvious characteristics of optical parts have solar panels and satellite body. Solar panels in the reflection direction of lightness value peak, once the angular luminance value will decrease rapidly, the satellite's body in every angle of the radiance values are larger. On the sunny side of the satellite mounting body mounted solar panels, absorb light, solar energy conversion; on the shady side of japanning, earth and other celestial bodies reflection absorption of stray light, effectively reduce the size of the satellite. Effective design of the head of the satellite body can also to some extent to achieve stealth effect. In a certain volume, satellite body are designed into the spherical, tapered, cubes and cylinders, the shape of the main body as shown in Figure 3.

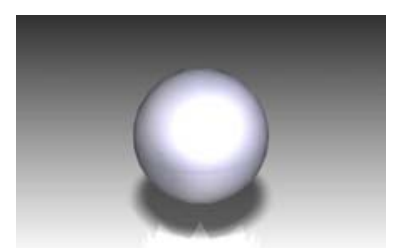

(a)spherical

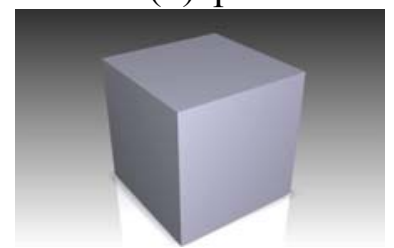

(c) cubes

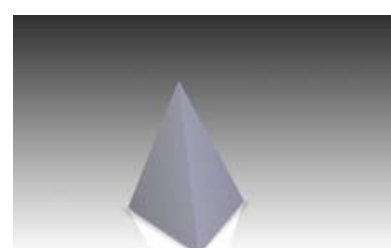

(b)tapered

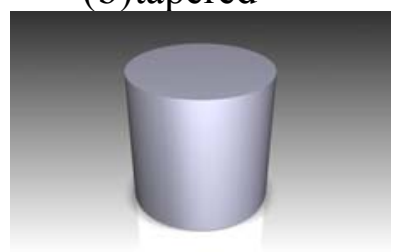

(d)cylinders

Figure 3 .satellite ontology model

The BRDF of the visible light band of the target surface material is $f_{\mathrm{r}}=\frac{1}{\pi}$, model volume was $1 \mathrm{~m}^{3}$, Target model parameters are: the radius of the sphere $0.62 \mathrm{~m}$; at the bottom of the cone surface to the bottom of $2 \mathrm{~m}, 1.5 \mathrm{~m}$ high isosceles triangle cone height of $2 \mathrm{~m}$; cube side length of $1 \mathrm{~m}$; the bottom surface of the cylinder radius $0.5 \mathrm{~m}$, high $1 \mathrm{~m}$.

The top of the satellite is always aligned with the sun, and the detector is observed around the satellite, the solar phase angle changed from $0^{\circ}$ to $180^{\circ}$, and the interval was $2^{\circ}$.

Table 1 maximum OCS value of each model

\begin{tabular}{|c|c|c|c|c|}
\hline model & spherical & cubes & cylinders & tapered \\
\hline $\begin{array}{c}\text { Max } \\
\text { OCS }\left(\mathrm{m}^{2}\right)\end{array}$ & 0.118 & 0.159 & 0.124 & 0.075 \\
\hline
\end{tabular}



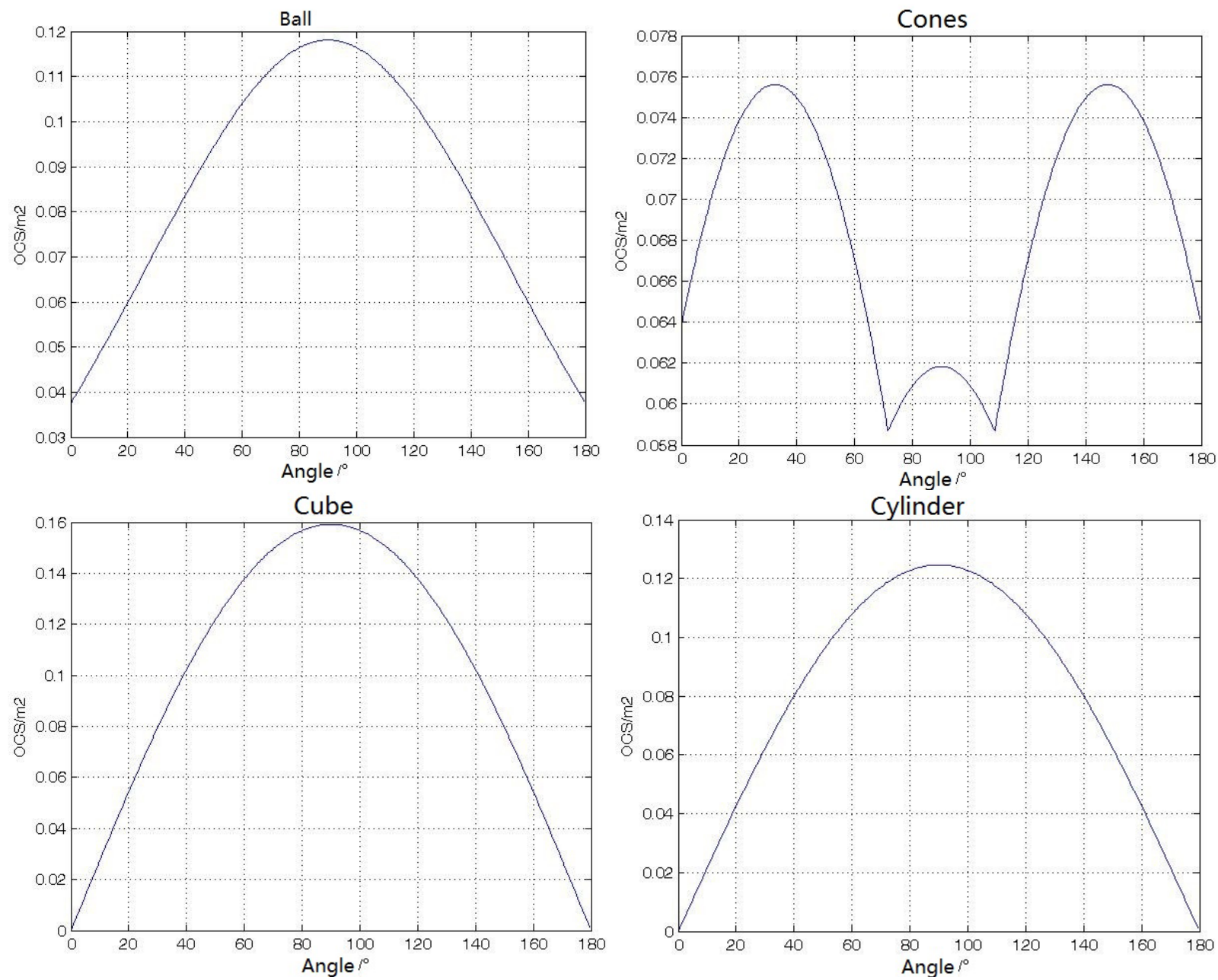

Fig. 4 Target satellite OCS variation curve

By comparing the above, it is concluded that, under the same volume, the main body of the satellite is designed to be triangular pyramid, and the maximum OCS value is the smallest in the four shapes. In the same case, the radiation brightness of the triangular pyramid is the smallest, the observed star will receive less light, thus reducing the probability of the detector to find the target star. The satellite body is designed into a triangular pyramid, and its stealth effect is the best in the four shapes.

\section{Summary}

In this paper, we first introduce the high earth orbit satellite, which is facing a lot of space surveillance system, which is the biggest threat to optical detection. Secondly, analyzes the present situation of the Space-Based Surveillance System and visibility of space target. Finally from the exterior design, material selection and processing the occlusion interference etc. of stealth satellite optical analysis, in a certain extent reduce satellite optical properties, for solar panels and satellite body shape design were proposed to reduce the brightness value, so as to achieve the purpose of invisibility. But the satellite optical stealth technology is complex, difficult, involving many disciplines, but also to meet the thermal control, the normal operation of the load and so on. It is a complicated project to realize the true optical stealth of the satellite and also needs to invest more manpower and material resources to study.

\section{References}

[1] Chen Jie, Pan feng, Su Tongling. The space-based space surveillance system [J]. national defense science and technology, 2011, 32 (1): 67-70.

[2] Wang Caiyun, Liao Wenhe. The present situation of stealth satellites and their application in space attack and defense [J]. aerospace electronic warfare, 2011, 27 (4): 17-19. 
[3] Li Yu Bo, Lu Yuan, Ling Yongshun. Optical characteristics of satellites and stealth on [J]. Journal of atmospheric and Environmental Optics, 2004, 17 (4): 63-65.

[4] Yang Zheng. The United States is about to launch the SBSS satellite [J]. foreign nuclear weapon dynamic reference, 2010, (2).

[5] Tan Ying. Study on space electronic technology[J], technology of space target detection 2006, 3 (3): 5-9.

[6] Li Yanbin, Jiang Lizhong, Huang Yong. Study on the development of the space target detection and surveillance system [J]. guidance and fuze, 2012, 33 (3): 50-60.

[7] Qiao Kai, Wang Zhile, Cong Mingyu. Space-based and ground-based surveillance system and compared analysis [J]. Optical technique, 2006, 32 (5): 744-746.

[8] Li Ying, Zhang Zhanyue, Fang Xiuhua. Development status and Prospect of space target surveillance system [J]. international space, 2004 (6): 28-32.

[9] Lin Bo. Technical research on the characteristics of low radiation / low optical brightness of micro satellite [D]. Nanjing University of Aeronautics \&amp; Astronautics, 2009

[10] Lin Laixing. Journal of foreign micro satellite in space and Research on the application of [J]. command and technology of equipment 2006, 17 (6): 47-49.

[11] Geng Kai. Mechanical Engineer [J]. numerical simulation the surface structure of solar cell, 2013 (10): 39-41.

[12] Zhou Changling, Wei Meiling, Yubaida, et al. stealth technology and modern technology of ceramic materials overview [J]. 2003, 24 (4): 23-27.

[13] Hao Yuncai, Xiao Shuqin, Wang Lixia. Current situation and development of optical remote sensing technology for spaceborne optical remote sensor $[\mathrm{J}]$. China Space Science and technology, 1995 (3): 40-50. 